\title{
THE STABILITY OF COLLOCATION METHODS FOR VIDES OF SECOND ORDER
}

\author{
EDRIS RAWASHDEH, DAVE MCDOWELL, AND LEELA RAKESH
}

Received 8 August 2004 and in revised form 29 December 2004

Simplest results presented here are the stability criteria of collocation methods for the second-order Volterra integrodifferential equation (VIDE) by polynomial spline functions. The polynomial spline collocation method is stable if all eigenvalues of a matrix are in the unit disk and all eigenvalues with $|\lambda|=1$ belong to a $1 \times 1$ Jordan block. Also many other conditions are derived depending upon the choice of collocation parameters used in the solution procedure.

\section{Introduction}

In order to discuss the numerical stability, we consider the linear second-order Volterra integrodifferential equation of the form

$$
y^{(2)}(t)=q(t)+\sum_{i=0}^{1} p_{i}(t) y^{(i)}(t)+\sum_{i=0}^{1} \int_{0}^{t} k_{i}(t, s) y^{(i)}(s) d s, \quad t \in I:=[0, T]
$$

with

$$
y(0)=y_{0}, \quad y^{(1)}(0)=y_{1}
$$

where $q: I \rightarrow R, p_{i}: I \rightarrow R$, and $k_{i}: D \rightarrow R(i=0,1)$ (with $D:=\{(t, s): 0 \leq s \leq t \leq T\}$ ) are given functions and are assumed to be (at least) continuous in the respective domains. For more details of these equations, many other interesting methods for the approximated solution and stability procedure are available in earlier literature $[1,2,3,4,5,6,9,10$, $12,13]$. The above equation is usually known as basis test equation and is suggested by Brunner and Lambert in [4]. Since then, it has been widely used for analyzing the stability properties of various methods.

Second-order VIDEs of the above form (1.1) will be solved numerically using polynomial spline spaces. In order to describe these approximating polynomial spline spaces, 
let $\prod_{N}: 0=t_{0}<t_{1}<\cdots<t_{N}=T$ be the mesh for the interval $I$, and set

$$
\begin{array}{cc}
\sigma_{n}:=\left[t_{n}, t_{n+1}\right], \quad h_{n}:=t_{n+1}-t_{n}, & n=0,1, \ldots, N-1, \\
h & =\max \left\{h_{n}: 0 \leq n \leq N-1\right\}, \quad \text { (mesh diameter), } \\
Z_{N}:=\left\{t_{n}: n=1,2, \ldots, N-1\right\}, & \bar{Z}_{N}=Z_{N} \cup\{T\} .
\end{array}
$$

Let $\pi_{m+d}$ be the set of (real) polynomials of degree not exceeding $m+d$, where $m \geq 1$ and $d \geq-1$ are given integers. The solution to initial-value problem (1.1) will be approximated by an element $u$ in the polynomial spline space,

$$
\begin{aligned}
& S_{m+d}^{(d)}\left(Z_{N}\right):=\left\{u:=\left.u(t)\right|_{t \in \sigma_{n}}:=u_{n}(t) \in \pi_{m+d}, n=0,1, \ldots, N-1,\right. \\
&\left.u_{n-1}^{(j)}\left(t_{n}\right)=u_{n}^{(j)}\left(t_{n}\right) \text { for } j=0,1, \ldots, d, t_{n} \in Z_{N}\right\} ;
\end{aligned}
$$

that is, by a polynomial spline function of degree $m+d$ which possesses the knots $Z_{N}$ and is $d$ times continuously differentiable on $I$. If $d=-1$, then the elements of $S_{m-1}^{(-1)}\left(Z_{N}\right)$ may have jump discontinuities at the knots $Z_{N}$. There are many other papers which had treated such problem using $S_{m}^{(0)}\left(Z_{N}\right)$ and $S_{m}^{(1)}\left(Z_{N}\right)[3,4,6]$ polynomial spline spaces.

According to Miculá et al. [11], an element $u \in S_{m+d}^{(d)}\left(Z_{N}\right)$ has the following form (for all $n=0,1, \ldots, N-1$ and $\left.t \in \sigma_{n}\right)$ :

$$
u(t)=u_{n}(t)=\sum_{r=0}^{d} \frac{u_{n-1}^{(r)}\left(t_{n}\right)}{r !}\left(t-t_{n}\right)^{r}+\sum_{r=1}^{m} a_{n, r}\left(t-t_{n}\right)^{d+r},
$$

where

$$
u_{-1}^{r}(0):=\left[\frac{d^{r}}{d t^{r}} u(t)\right]_{t=0}=y^{(r)}(0), \quad r=0,1, \ldots, d
$$

From (1.5), we see that the element $u \in S_{m+d}^{(d)}\left(Z_{N}\right)$ is well defined provided that the coefficients $\left\{a_{n, r}\right\}_{r=1, \ldots, m}$ for all $n=0,1, \ldots, N-1$ are known. In order to determine these coefficients, we consider a set of collocation parameters $\left\{c_{j}\right\}_{j=1, \ldots, m}$, where $0<c_{1}<\cdots<$ $c_{m} \leq 1$, and define the set of collocation points as

$$
X(N):=\bigcup_{n=0}^{N-1} X_{n}, \quad \text { with } X_{n}:=\left\{t_{n, j}:=t_{n}+c_{j} h_{n}, j=1,2, \ldots, m\right\} .
$$

The approximate solution $u \in S_{m+d}^{(d)}\left(Z_{N}\right)$ will be determined by imposing the condition that $u$ satisfies the initial-value problem $(1.1)$ on $X(N)$ and the initial conditions, that is,

$$
u^{(2)}(t)=q(t)+\sum_{i=0}^{1} p_{i}(t) u^{(i)}(t)+\sum_{i=0}^{1} \int_{0}^{t} k_{i}(t, s) u^{(i)}(s) d s, \quad \forall t \in X(N),
$$

with

$$
u(0)=y_{0}, \quad u^{(1)}(0)=y_{1}
$$

with a uniform mesh sequence $\left\{\prod_{N}\right\}, h_{n}=h$, for all $n=0,1, \ldots, N-1$. 


\section{Numerical stability}

In [7], Danciu studied the numerical stability of the collocation method for first-order integrodifferential equations. He studied the behavior of the method as applied to the initial-value problem integrodifferential test equation

$$
y^{\prime}(t)=q(t)+\alpha_{0} y(t)+v \int_{0}^{t} y(s) d s, \quad v \neq 0
$$

Equation (2.1) has been suggested by Brunner and Lambert in 1974 (see [4]), since then it has been extensively used as a basis for investigating the stability properties of several other methods.

In order to discuss the numerical stability for second-order integrodifferential equations, we study the numerical stability of the collocation spline method when applied to the initial-value problem integrodifferential test equation of the following form:

$$
\begin{gathered}
y^{\prime \prime}(t)=q(t)+\alpha_{0} y(t)+\alpha_{1} y^{\prime}(t)+v \int_{0}^{t} y(s) d s, \quad v \neq 0, \\
y(0)=y_{0}, \quad y^{\prime}(0)=y_{1},
\end{gathered}
$$

where $\alpha_{1}, \alpha_{2}$, and $\nu$ are constants, and the given function $g: I \rightarrow R$ is sufficiently smooth.

For simplicity, we use a polynomial spline collocation method in the space $S_{m+d}^{(d)}\left(Z_{N}\right)$, as an $(m, d)$-method (see [8]).

Definition 2.1. An $(m, d)$-method is said to be stable if all solutions $\left\{u\left(t_{n}\right)\right\}$ remain bounded, as $n \rightarrow \infty, h \rightarrow 0$ while $h_{n}$ remains fixed.

From (1.5), we observe that the first $d+1$ coefficients of the $u \in S_{m+d}^{(d)}\left(Z_{N}\right)$ are determine by the smooth conditions, and then the collocation conditions can be used to determine the last $m$ coefficients. Thus, for convenience, we introduce the following notations:

$$
\begin{gathered}
\eta_{n}:=\left(\eta_{n, r}\right)_{r=0, \ldots, d}, \quad \text { with } \eta_{n, r}:=\frac{u_{n-1}^{(r)}\left(t_{n}\right)}{r !} h^{r} ; \\
\beta_{n}:=\left(\beta_{n, r}\right)_{r=1, \ldots, m}, \quad \text { with } \beta_{n, r}:=a_{n, r} h^{d+r}, n=0,1, \ldots, N .
\end{gathered}
$$

Using (2.4) in (1.5), for all $t:=t_{n}+\tau h \in \sigma_{n}$, we have the following equation:

$$
u(t)=u_{n}\left(t_{n}+\tau h\right)=\sum_{r=0}^{d} \eta_{n, r} \tau^{r}+\sum_{r=1}^{m} \beta_{n, r} \tau^{d+r}, \quad \forall \tau \in(0,1], n=0,1, \ldots, N
$$

By applying the collocation method to test (2.2) for the case $d \geq 2$ and using (2.5) we have the following collocation equation:

$$
V \beta_{n}=W \eta_{n}+h^{2} R_{n}, \quad \forall n=0,1, \ldots, N-1,
$$




\section{Stability of the collocation methods}

where $V$ is the $m \times m$ matrix, $W$ is the $m \times(d+1)$ matrix, and $R_{n}$ is the $m$-vector, whose elements are given by

$$
\begin{aligned}
& V_{j, r}:=\left.(d+r)(d+r-1)-\alpha_{0} h^{2} c_{j}^{2}-\alpha_{1} h(d+r) c_{j}-\frac{v h^{3}}{(d+r+1)} c_{j}^{3}\right] c_{j}^{d+r-2}, \\
& W_{j, r}:= \begin{cases}\nu h^{3} c_{j} & \text { if } r=0, \\
\alpha_{0} h^{2} c_{j}+v \frac{h^{3}}{2} c_{j}^{2} & \text { if } r=1, \\
\alpha_{0} h^{2} c_{j}^{2}+2 \alpha_{1} h c_{j}+v \frac{h^{3}}{3} c_{j}^{3} & \text { if } r=2, \\
{\left[\alpha_{0} h^{2} c_{j}^{2}+\alpha_{1} h r c_{j}+v \frac{h^{3}}{r+1} c_{j}^{3}-r(r-1)\right] c_{j}^{r-2}} & \text { if } 3 \leq r \leq d,\end{cases} \\
& R_{n, j}:= \begin{cases}q\left(t_{0, j}\right)-q\left(t_{0}\right) & \text { if } n=0, \\
q\left(t_{n, j}\right)-q\left(t_{n-1, m}\right)+u_{n-1}^{\prime \prime}\left(t_{n-1, m}\right)-u_{n-1}^{\prime \prime}\left(t_{n}\right) & \\
+\alpha_{0}\left[u_{n-1}\left(t_{n}\right)-u_{n-1}\left(t_{n-1, m}\right)\right]+\alpha_{1}\left[u_{n-1}^{\prime}\left(t_{n}\right)-u_{n-1}^{\prime}\left(t_{n-1, m}\right)\right] & \text { if } n>0 . \\
+v h \int_{c_{m}}^{1} u_{n-1}\left(t_{n-1}+\tau h\right) d \tau & \end{cases}
\end{aligned}
$$

By direct differentiation of (2.5) and using the smooth conditions of the approximation $u \in S_{m+d}^{(d)}\left(Z_{N}\right)$, we get a relationship between vector $\eta_{n+1}$ and vectors $\eta_{n}$ and $\beta_{n}$, as follows:

$$
\eta_{n+1}=A \eta_{n}+B \beta_{n}, \quad \forall n=0,1, \ldots, N-1,
$$

where $A$ is the $(d+1) \times(d+1)$ upper triangular matrix and $B$ is the $(d+1) \times m$ matrix, whose elements are given by

$$
a_{j, r}:=\left\{\begin{array}{ll}
0 & \text { if } r<j, \\
\left(\begin{array}{l}
r \\
j
\end{array}\right) & \text { if } r \geq j,
\end{array} \quad b_{j, r}:=\left(\begin{array}{c}
d+r \\
j
\end{array}\right) .\right.
$$

For $h$ small enough, the matrix $V$ is invertible since the determinant of $V$ is a Vandermonde-type determinant for $h=0$. Hence from (2.6) and (2.8), we have

$$
\begin{aligned}
\eta_{n+1} & =A \eta_{n}+B V^{-1}\left[W \eta_{n}+h^{2} R_{n}\right] \\
& =\left(A+B V^{-1} W\right) \eta_{n}+h^{2} B V^{-1} R_{n} .
\end{aligned}
$$

Thus we have the following recurrence relation:

$$
\eta_{n+1}=M \eta_{n}+h^{2} B V^{-1} R_{n}
$$

where

$$
M:=A+B V^{-1} W
$$


Therefore, we have the following theorem which represents a stability criterion for the present method. The proof of this theorem is quite similar to the proof given by Danciu [7] for first-order VIDEs.

THeOREM 2.2. An $(m, d)$-method is stable if and only if all eigenvalues of matrix $M=A+$ $B V^{-1} W$ are in the unit disk and all eigenvalues with $|\lambda|=1$ belong to $a 1 \times 1$ Jordan block.

Remark 2.3. Note that the dimension of the matrix $M$ is $d+1$. Moreover, if we denote by $M_{0}$ the matrix $M$ with $h=0$, and by $\lambda^{(0)}$ and $\lambda$ the eigenvalues of $M_{0}$ and $M$, respectively, then it follows that the matrix $M_{0}$ has $\lambda_{1}^{(0)}=\lambda_{2}^{(0)}=\lambda_{3}^{(0)}=1$, for $m \geq 1$ and $d \geq 2$.

\section{Applications}

In this section, we will investigate the following special cases.

(I) For the case $d=2$, the approximation space is $S_{m+2}^{(2)}\left(Z_{N}\right)$. From Theorem 2.2 and Remark 2.3, we have the following theorem.

THeOREM 3.1. For every choice of the collocation parameters $\left\{c_{j}\right\}_{j=1, m}$, an $(m, 2)$-method is stable for all $m \geq 1$.

(II) For the case $m=1$, this choice of $m$ corresponds to a classical spline function, that is, the approximate solution $u \in S_{1+d}^{(d)}\left(Z_{N}\right)$. Using notations from Remark 2.3 (i.e., $M_{0}$ is the matrix $M$ with $h=0$, and $\lambda^{(0)}$ and $\lambda$ are the respective eigenvalues of $M_{0}$ and $M$ ), we have

$$
\lambda=\lambda^{(0)}+O(h)
$$

If $c_{1} \in(0,1]$ is the collocation parameter, then for all $d \geq 1$, using the binomial expansion, we find that for matrix $M_{0}$ the trace is,

$$
\operatorname{Tr}\left(M_{0}\right)=d+2+\frac{1}{c_{1}^{d-1}}-\left(1+\frac{1}{c_{1}}\right)^{d-1} .
$$

As regard the stability of the spline collocation method, we have the following result.

Theorem 3.2. A $(1, d)$-method is stable if and only if one of the following conditions is true:

(i) $d=2$ and $c_{1} \in(0,1]$,

(ii) $d=3$ and $c_{1}=1$.

Proof. For the case $d=2$, this theorem follows from Theorem 3.1. If $d=3$, then the fourth eigenvalue of $M_{0}$ is $\lambda_{4}^{(0)}=1-\left(2 / c_{1}\right) \leq-1$ for $c_{1} \in(0,1]$, and its absolute value is 1 , if and only if $c_{1}=1$. If $d \geq 4$, then setting $p=d-1$ in (3.2), we have

$$
\operatorname{Tr}\left(M_{0}\right)=p+3+\frac{1}{c_{1}^{p}}-\left(1+\frac{1}{c_{1}}\right)^{p}
$$

so if $d>4$ and $c_{1} \in(0,1]$, then

$$
\infty<\operatorname{Tr}\left(M_{0}\right)<-p=-(d-1)
$$




\section{Stability of the collocation methods}

Since $\operatorname{Tr}\left(M_{0}\right)=\lambda_{1}^{(0)}+\lambda_{2}^{(0)}+\cdots+\lambda_{d+1}^{(0)}<-d+1$, and $\lambda_{1}^{(0)}=1$, it results that there exists an eigenvalue $\lambda^{(0)}$ whose value is smaller than -1 . If $d=4$ then from (3.2), $\lambda_{4}^{(0)}+\lambda_{5}^{(0)} \leq-4$, and therefore $\lambda_{4}^{(0)}<-1$ or $\lambda_{5}^{(0)}<-1$. Thus from Theorem 2.2 we have that, for $d \geq 4$, a $(1, d)$-method is unstable for any choice of the collocation parameter $c_{1} \in(0,1]$.

(III) For $m=2$, we can prove the following result but the proof is the same as in [7].

Theorem 3.3. Let $0<c_{1}<c_{2} \leq 1$ be the collocation parameters, then

(i) a (2,2)-method is stable for every choice of the collocation parameters,

(ii) a (2,3)-method is stable if and only if $c_{1}+c_{2} \geq 3 / 2$,

(iii) if $c_{2}=1$, then $a(2, d)$-method is unstable for all $d \geq 4$.

(IV) For the case $d=3$, the approximation $u \in S_{m+3}^{(3)}\left(Z_{N}\right)$ and the dimension of the matrix $M_{0}$ is 4 and its first $p+1$ eigenvalues are $\lambda_{1}^{(0)}=\lambda_{2}^{(0)}=\lambda_{3}^{(0)}=1$. To compute the fourth eigenvalue, we need the following results. But, first we introduce the following notations:

$$
\begin{aligned}
& S_{k}:=S_{k}\left(c_{1}, \ldots, c_{m}\right)=\sum_{1 \leq i_{1}<\cdots<i_{k} \leq m}^{m} c_{i_{1}} c_{i_{2}} \cdots c_{i_{k}}, \quad \text { for } 1 \leq k \leq m \text {, } \\
& S_{0}:=S_{0}\left(c_{1}, \ldots, c_{m}\right)=1 \text {, } \\
& S_{k, j}:=S_{k}\left(c_{1}, \ldots, c_{j-1}, c_{j+1}, \ldots, c_{m}\right), \quad \text { for } 1 \leq k \leq m-1,1 \leq j \leq m \text {. }
\end{aligned}
$$

Lemma 3.4. Let $0<c_{1}<c_{2}<\cdots<c_{m} \leq 1$ be the collocation parameters, then

$$
\left|\begin{array}{cccccccc}
1 & c_{1} & c_{1}^{2} & \cdots & c_{1}^{i-1} & c_{1}^{i+1} & \cdots & c_{1}^{m} \\
1 & c_{2} & c_{2}^{2} & \cdots & c_{2}^{i-1} & c_{2}^{i+1} & \cdots & c_{2}^{m} \\
\vdots & \vdots & \vdots & \vdots & \vdots & \vdots & \vdots & \vdots \\
1 & c_{m} & c_{m}^{2} & \cdots & c_{m}^{i-1} & c_{m}^{i+1} & \cdots & c_{m}^{m}
\end{array}\right|=S_{m-i} \prod_{1 \leq k<j \leq m}^{m}\left(c_{j}-c_{k}\right)
$$

Proof. We will prove the lemma by induction on the dimension of the matrix, starting with $2 \times 2$ matrices. For the $2 \times 2$ matrices, the result is clearly true. For $m \times m$ matrices $(m>2)$, we define

$$
f(x):=\left|\begin{array}{cccccccc}
1 & c_{1} & c_{1}^{2} & \cdots & c_{1}^{i-1} & c_{1}^{i+1} & \cdots & c_{1}^{m} \\
1 & c_{2} & c_{2}^{2} & \cdots & c_{2}^{i-1} & c_{2}^{i+1} & \cdots & c_{2}^{m} \\
\vdots & \vdots & \vdots & \vdots & \vdots & \vdots & \vdots & \vdots \\
1 & c_{m-1} & c_{m-1}^{2} & \cdots & c_{m-1}^{i-1} & c_{m-1}^{i+1} & \cdots & c_{m-1}^{m} \\
1 & x & x^{2} & \cdots & x^{i-1} & x^{i+1} & \cdots & x^{m}
\end{array}\right| .
$$


Note that

$$
\left|\begin{array}{cccccccc}
1 & c_{1} & c_{1}^{2} & \cdots & c_{1}^{i-1} & c_{1}^{i+1} & \cdots & c_{1}^{m} \\
1 & c_{2} & c_{2}^{2} & \cdots & c_{2}^{i-1} & c_{2}^{i+1} & \cdots & c_{2}^{m} \\
\vdots & \vdots & \vdots & \vdots & \vdots & \vdots & \vdots & \vdots \\
1 & c_{m} & c_{m}^{2} & \cdots & c_{m}^{i-1} & c_{m}^{i+1} & \cdots & c_{m}^{m}
\end{array}\right|=f\left(c_{m}\right) .
$$

Now, since $f\left(c_{1}\right)=f\left(c_{2}\right)=\cdots=f\left(c_{m-1}\right)=0$, we have

$$
f(x)=a(x-b) \prod_{i=1}^{m-1}\left(x-c_{i}\right),
$$

where $a, b$ are constants to be determined. By the induction hypothesis, we obtain

$$
a=S_{m-1-i}\left(c_{1}, \ldots, c_{m-1}\right) \prod_{k<j}^{m-1}\left(c_{j}-c_{k}\right) .
$$

Moreover, from (3.9),

$$
f(0)=a(-1)^{m} c_{1} c_{2} \cdots c_{m-1} b .
$$

On the other hand, from the definition of $f$ and by the induction hypothesis, we have

$$
\begin{aligned}
f(0) & =(-1)^{m+1}\left|\begin{array}{ccccccc}
c_{1} & c_{1}^{2} & \cdots & c_{1}^{i-1} & c_{1}^{i+1} & \cdots & c_{1}^{m} \\
c_{2} & c_{2}^{2} & \cdots & c_{2}^{i-1} & c_{2}^{i+1} & \cdots & c_{2}^{m} \\
\vdots & \vdots & \vdots & \vdots & \vdots & \vdots & \vdots \\
c_{m-1} & c_{m-1}^{2} & \cdots & c_{m-1}^{i-1} & c_{m-1}^{i+1} & \cdots & c_{m-1}^{m}
\end{array}\right| \\
& =(-1)^{m+1} c_{1} c_{2} \cdots c_{m-1} S_{m-i}\left(c_{1}, \ldots, c_{m-1}\right) \prod_{k<j}^{m-1}\left(c_{j}-c_{k}\right) .
\end{aligned}
$$

Thus, from (3.11) and (3.12), we have

$$
-a b=S_{m-i}\left(c_{1}, \ldots, c_{m-1}\right) \prod_{k<j}^{m-1}\left(c_{j}-c_{k}\right),
$$

and so

$$
\begin{aligned}
f\left(c_{m}\right)= & a\left(c_{m}-b\right) \prod_{i=1}^{m-1}\left(c_{m}-c_{i}\right) \\
= & {\left[c_{m} S_{m-1-i}\left(c_{1}, \ldots, c_{m-1}\right) \prod_{k<j}^{m-1}\left(c_{j}-c_{k}\right)\right.} \\
& \left.+S_{m-i}\left(c_{1}, \ldots, c_{m-1}\right) \prod_{k<j}^{m-1}\left(c_{j}-c_{k}\right)\right] \prod_{i=1}^{m-1}\left(c_{m}-c_{i}\right) .
\end{aligned}
$$


1056 Stability of the collocation methods

However, since

$$
\begin{gathered}
c_{m} S_{m-1-i}\left(c_{1}, \ldots, c_{m-1}\right)+S_{m-i}\left(c_{1}, \ldots, c_{m-1}\right)=S_{m-i}\left(c_{1}, \ldots, c_{m}\right)=S_{m-i} \\
\prod_{k<j}^{m-1}\left(c_{j}-c_{k}\right) \prod_{i=1}^{m-1}\left(c_{m}-c_{i}\right)=\prod_{k<j}^{m}\left(c_{j}-c_{k}\right)
\end{gathered}
$$

we have

$$
f\left(c_{m}\right)=S_{m-i} \prod_{k<j}^{m}\left(c_{j}-c_{k}\right)
$$

which proves the lemma.

Remark 3.5. Note that in Lemma 3.4, if $i=m$, then we have the Vandermonde determinant.

Corollary 3.6. Let $V_{0}$ be the matrix $V$ with $h=0, d=3$, that is, $V_{0}$ is the $m \times m$ matrix whose elements are

$$
\left(V_{0}\right)_{j, r}:=((r+3)(r+2)) C_{j}^{r+1}
$$

Then, $V_{0}^{-1}$ is the matrix whose elements are

$$
\left(V_{0}^{-1}\right)_{r, j}=\frac{1}{\operatorname{det}\left(V_{0}\right)}(-1)^{r+j} S_{m-1, j}^{2} S_{m-r, j} \prod_{l<k,(l, k \neq j)}\left(c_{k}-c_{l}\right) \prod_{k=1,(k \neq r)}^{m}(k+2)(k+3),
$$

where

$$
\operatorname{det}\left(V_{0}\right)=\left[\prod_{k=1}^{m}(k+2)(k+3) \prod_{l<k}\left(c_{k}-c_{l}\right)\right] S_{m}^{2}
$$

Proof. From Lemma 3.4, we have

$$
\operatorname{det}\left(V_{0}\right)=\left[\prod_{k=1}^{m}(k+2)(k+3) \prod_{l<k}\left(c_{k}-c_{l}\right)\right] S_{m}^{2}
$$

Now

$$
V_{0}^{-1}=\frac{\operatorname{Adj}\left(V_{0}\right)}{\operatorname{det}\left(V_{0}\right)}
$$


where $\operatorname{Adj}\left(V_{0}\right)$ is the adjoint matrix of $V_{0}$, however,

$$
\begin{aligned}
\operatorname{Adj}\left(V_{0}\right)_{r, j}= & (-1)^{r+j} S_{m-1, j}^{2} \prod_{k=1,(k \neq r)}^{m}(k+2)(k+3) \\
& \times\left|\begin{array}{cccccccc}
1 & c_{1} & c_{1}^{2} & \cdots & c_{1}^{r-2} & c_{1}^{r} & \cdots & c_{1}^{m-1} \\
1 & c_{2} & c_{2}^{2} & \cdots & c_{2}^{r-2} & c_{2}^{r} & \cdots & c_{2}^{m-1} \\
\vdots & \vdots & \vdots & \vdots & \vdots & \vdots & \vdots & \vdots \\
1 & c_{j-1} & c_{j-1}^{2} & \cdots & c_{j-1}^{r-2} & c_{j-1}^{r} & \cdots & c_{j-1}^{m-1} \\
1 & c_{j+1} & c_{j+1}^{2} & \cdots & c_{j+1}^{r-2} & c_{j+1}^{r} & \cdots & c_{j+1}^{m-1} \\
\vdots & \vdots & \vdots & \vdots & \vdots & \vdots & \vdots & \vdots \\
1 & c_{m} & c_{m}^{2} & \cdots & c_{m}^{r-2} & c_{m}^{r} & \cdots & c_{m}^{m-1}
\end{array}\right| .
\end{aligned}
$$

Again, by Lemma 3.4 and using relations

$$
S_{m-1-(r-1)}\left(c_{1}, \ldots, c_{j-1}, c_{j+1}, \ldots, c_{m}\right)=S_{m-r}\left(c_{1}, \ldots, c_{j-1}, c_{j+1}, \ldots, c_{m}\right)=S_{m-r, j},
$$

we have

$$
\left|\begin{array}{cccccccc}
1 & c_{1} & c_{1}^{2} & \cdots & c_{1}^{r-2} & c_{1}^{r} & \cdots & c_{1}^{m-1} \\
1 & c_{2} & c_{2}^{2} & \cdots & c_{2}^{r-2} & c_{2}^{r} & \cdots & c_{2}^{m-1} \\
\vdots & \vdots & \vdots & \vdots & \vdots & \vdots & \vdots & \vdots \\
1 & c_{j-1} & c_{j-1}^{2} & \cdots & c_{j-1}^{r-2} & c_{j-1}^{r} & \cdots & c_{j-1}^{m-1} \\
1 & c_{j+1} & c_{j+1}^{2} & \cdots & c_{j+1}^{r-2} & c_{j+1}^{r} & \cdots & c_{j+1}^{m-1} \\
\vdots & \vdots & \vdots & \vdots & \vdots & \vdots & \vdots & \vdots \\
1 & c_{m} & c_{m}^{2} & \cdots & c_{m}^{r-2} & c_{m}^{r} & \cdots & c_{m}^{m-1}
\end{array}\right|=S_{m-r, j} \prod_{l<k,(l, k \neq j)}^{m}\left(c_{k}-c_{l}\right) .
$$

Thus,

$$
\left(V_{0}^{-1}\right)_{r, j}=\frac{1}{\operatorname{det}\left(V_{0}\right)}(-1)^{r+j} S_{m-1, j}^{2} S_{m-r, j} \prod_{l<k,(l, k \neq j)}\left(c_{k}-c_{l}\right) \prod_{k=1,(k \neq r)}^{m}(k+2)(k+3),
$$

which completes the proof of the corollary.

Now, we can derive a formula for computing the $p+2$-eigenvalue of the matrix $M_{0}$.

Theorem 3.7. For the case $d=3$ and $m \geq 1$, the $p+2$-eigenvalue of $M_{0}$ can be computed by using the following relation:

$$
\lambda_{4}^{(0)}=\frac{S_{m}-2 S_{m-1}+3 S_{m-2}+\cdots+(-1)^{m-1} m S_{1}+(-1)^{m}(m+1)}{S_{m}} .
$$


1058 Stability of the collocation methods

Proof. Let $V_{0}$ and $W_{0}$ be the matrices $V$ and $W$, respectively, with $h=0$, then for $d=3$, $W_{0}$ is an $m \times 4$ matrix whose elements are given by

$$
\left(W_{0}\right)_{j, r}:= \begin{cases}0 & \text { if } r=0,1,2 \\ -6 c_{j} & \text { if } r=3\end{cases}
$$

Now, the fourth eigenvalue of $M_{0}=A+B V_{0}^{-1} W_{0}$ is

$$
\lambda_{4}^{(0)}=1+\sum_{r=1}^{m}(B)_{4, r}\left(V_{0}^{-1} W_{0}\right)_{r, 4}
$$

From (2.8), the entries of the last row of matrix $B$ are

$$
(B)_{4, r}=\left(\begin{array}{c}
3+r \\
3
\end{array}\right)
$$

Moreover, from (3.27) and Corollary 3.6, we have

$$
\begin{aligned}
\left(V_{0}^{-1} W_{0}\right)_{r, 4}=\frac{-6}{\operatorname{det}\left(V_{0}\right)} \sum_{j=1}^{m} & {\left[(-1)^{(r+j)} S_{m-1, j}^{2} S_{m-r, j} c_{j}\right.} \\
& \left.\times \prod_{l<k,(l, k \neq j)}\left(c_{k}-c_{l}\right) \prod_{k=1,(k \neq r)}^{m}(k+2)(k+3)\right] .
\end{aligned}
$$

Therefore,

$$
\begin{aligned}
\lambda_{4}^{(0)}=1+\frac{6}{\operatorname{det}\left(V_{0}\right)} \sum_{r=1}^{m} \sum_{j=1}^{m} & {\left[\left(\begin{array}{c}
3+r \\
3
\end{array}\right)(-1)^{(r+j+1)} S_{m-1, j}^{2} S_{m-r, j} c_{j}\right.} \\
& \left.\times \prod_{k<k,(l, k \neq j)}\left(c_{k}-c_{l}\right) \prod_{k=1,(k \neq r)}^{m}(k+2)(k+3)\right] .
\end{aligned}
$$

By using relations

$$
\begin{gathered}
c_{j} S_{m-1, j}^{2}=S_{m} S_{m-1, j}, \\
6\left(\begin{array}{c}
3+r \\
3
\end{array}\right) \prod_{k=1,(k \neq r)}^{m}(k+2)(k+3)=(r+1) \prod_{k=1}^{m}(k+2)(k+3),
\end{gathered}
$$

and $\operatorname{det}\left(V_{0}\right)$, the above expression can be simplified as follows:

$$
\lambda_{4}^{(0)}=1+\frac{\sum_{r=1}^{m}(-1)^{r}(r+1) \sum_{j=1}^{m}(-1)^{(j+1)} S_{m-1, j} S_{m-r, j} \prod_{l<k,(l, k \neq j)}\left(c_{k}-c_{l}\right)}{S_{m} \prod_{l<k}\left(c_{k}-c_{l}\right)} .
$$


However, from Lemma 3.4, we have

$$
\begin{aligned}
\sum_{j=1}^{m}(-1)^{(j+1)} S_{m-1, j} S_{m-r, j} \prod_{l<k,(l, k \neq j)}\left(c_{k}-c_{l}\right) & =\left|\begin{array}{cccccccc}
1 & c_{1} & c_{1}^{2} & \cdots & c_{1}^{r-1} & c_{1}^{r+1} & \cdots & c_{1}^{m} \\
1 & c_{2} & c_{2}^{2} & \cdots & c_{2}^{r-1} & c_{2}^{r+1} & \cdots & c_{2}^{m} \\
\vdots & \vdots & \vdots & \vdots & \vdots & \vdots & \vdots & \vdots \\
1 & c_{m} & c_{m}^{2} & \cdots & c_{m}^{r-1} & c_{m}^{r+1} & \cdots & c_{m}^{m}
\end{array}\right| \\
& =S_{m-r} \prod_{l<k}^{m}\left(c_{k}-c_{l}\right) .
\end{aligned}
$$

Hence,

$$
\begin{aligned}
\lambda_{p+2}^{(0)} & =1+\frac{\sum_{r=1}^{m}(-1)^{r}(r+1) S_{m-r}}{S_{m}} \\
& =\frac{\sum_{r=0}^{m}(-1)^{r}(r+1) S_{m-r}}{S_{m}} \\
& =\frac{S_{m}-2 S_{m-1}+3 S_{m-2}+\cdots+(-1)^{m-1} m S_{1}+(-1)^{m}(m+1)}{S_{m}},
\end{aligned}
$$

which concludes the proof of Theorem 3.7.

Remark 3.8. Theorem 3.7 proves the conjecture asserted by Danciu [7] for first-order integrodifferential equations $(p=1, d=2)$.

As an application to Theorem 3.7, we can prove the following results. The proofs are quite similar to [7] for the first-order Volterra integrodifferential equation.

Corollary 3.9. An $(m, 3)$-method is stable if and only if

$$
\left|\frac{\left[d / d t\left(t \cdot R_{m}(t)\right)\right]_{t=1}}{R_{m}(0)}\right| \leq 1,
$$

where $R_{m}(t)$ is the polynomial of degree $m$ whose zeroes are the collocation parameters $\left\{c_{j}\right\}_{j=1, \ldots, m}$.

Regarding the stability of local superconvergent solution $u \in S_{m+4}^{(3)}\left(Z_{n}\right)$, we have the following corollary.

Corollary 3.10. (i) If the collocation parameters $\left\{c_{j}\right\}_{j=1, \ldots, m}$ are uniformly distributed in $(0,1]$ (i.e., $c_{j}=j / m$, for all $\left.j=1,2, \ldots, m\right)$, then $(m, 3)$-method is stable for $m \geq 1$.

(ii) If the collocation parameters $\left\{c_{j}\right\}_{j=1, \ldots, m}$ are the Radau II points in the interval $(0,1]$, then ( $m, 3)$-method is unstable for $m \geq 2$.

(iii) If the collocation parameters $\left\{c_{j}\right\}_{j=1, \ldots, m}$ are the Gauss points in the interval $(0,1]$, then $(m, 3)$-method is unstable for $m \geq 2$.

(iv) If the first $m-1$ collocation parameters $\left\{c_{j}\right\}_{j=1, \ldots, m}$ are the Gauss points in the interval $(0,1)$ and the last collocation parameter is $c_{m}=1$, then $(m, 3)$-method is stable for $m \geq 2$. 
1060 Stability of the collocation methods

\section{Stability of $S_{m}^{(0)}\left(Z_{n}\right)$}

In this section, we will investigate the stability when $d=0$.

From (2.5), the restriction of $u \in S_{m}^{(0)}\left(Z_{n}\right)$ to the subinterval $\sigma_{n}$ is given by

$$
u(t)=u_{n}\left(t_{n}+\tau h\right)=u_{n-1}\left(t_{n}\right)+\sum_{r=1}^{m} \beta_{n, r} \tau^{r}, \quad \text { for } \tau \in(0,1], n=0,1, \ldots, N-1 .
$$

If we denote by $u_{n+1}$ and by $u_{n+1}^{\prime \prime}$ the vectors with $m$ elements

$$
u_{n+1}:=\left(u_{n}\left(t_{n}+c_{j} h\right)\right)_{j=1, \ldots m}^{T}, \quad u_{n+1}^{\prime \prime}:=\left(u_{n}^{\prime \prime}\left(t_{n}+c_{j} h\right)\right)_{j=1, \ldots m}^{T},
$$

then from (4.1), we obtain

$$
\begin{aligned}
& u_{n+1}=(1,1, \ldots, 1)^{T} u_{n-1}\left(t_{n}\right)+E \beta_{n}, \quad \text { for } n=0,1, \ldots, N-1, \\
& u_{n+1}^{\prime \prime}=h^{-2} E^{\prime \prime} \beta_{n}, \quad \text { for } n=0,1, \ldots, N-1 .
\end{aligned}
$$

Here the matrices $E$ and $E^{\prime \prime}$ are $m \times m$ matrices defined by $E:=\left(c_{j}^{r}\right)_{j, r=1, \ldots, m}$ and $E^{\prime \prime}:=$ $\left(r(r-1) c_{j}^{r-2}\right)_{j, r=1, \ldots, m}$.

In this case, the collocation equation becomes

$$
V \beta_{n}=h^{2} W^{\prime}\left(u_{n-1}\left(t_{n}\right), u_{n-1}^{\prime}\left(t_{n}\right), u_{n-1}^{\prime \prime}\left(t_{n}\right)\right)^{T}+h^{2} R_{n}
$$

for $n=0,1 \ldots, N-1$, where $W^{\prime}$ is the $m \times 3$ matrix whose elements are

$$
\left(W^{\prime}\right)_{j, r}:= \begin{cases}\nu h c_{j} & \text { if } r=1 \\ -\alpha_{1} & \text { if } r=2 \\ 1 & \text { if } r=3\end{cases}
$$

and the matrix $V$ and the vector $R_{n}$ are defined in (2.6) when $d=0$.

Since $V=E^{\prime \prime}+O(h)$, the elimination of $\beta_{n}$ between (4.4) and (4.5) yields

$$
\begin{aligned}
u_{n}^{\prime \prime}\left(t_{n, j}\right)= & (1+O(h)) u_{n-1}^{\prime \prime}\left(t_{n}\right)+(1+O(h)) u_{n-1}^{\prime}\left(t_{n}\right) \\
& +O(h) u_{n-1}\left(t_{n}\right)+(1+O(h)) R_{n, j}, \\
& \text { for } j=1,2, \ldots, m \quad(n=0,1, \ldots, N-1) .
\end{aligned}
$$

For $\tau \in[0,1]$, the second derivatives of the approximation $u \in S_{m}^{(0)}\left(Z_{n}\right)$ may be written in the form

$$
u_{n}^{\prime \prime}(t+\tau h)=\sum_{j=1}^{m} L_{j}(\tau) u_{n}^{\prime \prime}\left(t_{n, j}\right), \quad \text { for } n=0,1, \ldots, N-1
$$

where

$$
L_{j}(\tau):=\prod_{j=1, i \neq j}^{m} \frac{\left(\tau-c_{i}\right)}{\left(c_{j}-c_{i}\right)}, \quad \text { for } j=0,1, \ldots, m
$$


are the Lagrange fundamental polynomials associated with the collocation parameters $\left\{c_{j}\right\}_{j=1, m}$. Now, replacing $u^{\prime \prime}\left(t_{n, j}\right)$ in (4.8) with its values given by (4.7), for $n=0,1, \ldots$, $N-1$, we obtain

$$
\begin{aligned}
u_{n}^{\prime \prime}\left(t_{n+1}\right)= & (1+O(h))\left(u_{n-1}^{\prime \prime}\left(t_{n}\right)+u_{n-1}^{\prime}\left(t_{n}\right)+\sum_{j=1}^{m} L_{j}(1) R_{n, j}\right) \\
& +O(h) u_{n-1}\left(t_{n}\right), \quad \text { for } j=1,2, \ldots, m \quad(n=0,1, \ldots, N-1) .
\end{aligned}
$$

By integrating relation (4.8), for $\tau \in[0,1]$, and using relation (4.7), we obtain

$$
\begin{aligned}
u_{n}^{\prime}\left(t_{n+1}\right)= & h(1+O(h)) u_{n-1}^{\prime \prime}\left(t_{n}\right)+(1+h(1+O(h))) u_{n-1}^{\prime}\left(t_{n}\right) \\
& +h O(h) u_{n-1}\left(t_{n}\right)+h(1+O(h)) \int_{0}^{1}\left(\sum_{j=1}^{m} L_{j}(\tau) R_{n, j}\right) d \tau, \\
& \text { for } j=1,2, \ldots, m \quad(n=0,1, \ldots, N-1) .
\end{aligned}
$$

Integrating (4.8) one more time and using relation (4.7) yields

$$
\begin{aligned}
u_{n}\left(t_{n+1}\right)= & h^{2}(1+O(h))\left(u_{n-1}^{\prime \prime}\left(t_{n}\right)+u_{n-1}^{\prime}\left(t_{n}\right)\right)+\left(1+h^{2} O(h)\right) u_{n-1}\left(t_{n}\right) \\
& +h^{2}(1+O(h)) \int_{0}^{1} \int_{0}^{s}\left(\sum_{j=1}^{m} L_{j}(\tau) R_{n, j}\right) d \tau d s, \\
& \text { for } j=1,2, \ldots, m \quad(n=0,1, \ldots, N-1) .
\end{aligned}
$$

Equations (4.7), (4.11), and (4.12) together form a system which may be written in the form

$$
\left(\begin{array}{l}
u_{n}\left(t_{n+1}\right) \\
u_{n}^{\prime}\left(t_{n+1}\right) \\
u_{n}^{\prime \prime}\left(t_{n+1}\right)
\end{array}\right)=M^{\prime}\left(\begin{array}{l}
u_{n-1}\left(t_{n}\right) \\
u_{n-1}^{\prime}\left(t_{n}\right) \\
u_{n-1}^{\prime \prime}\left(t_{n}\right)
\end{array}\right)+(1+O(h)) R^{\prime}{ }_{n} \quad \text { for } n=0,1, \ldots, N-1,
$$

where

$$
\begin{array}{r}
M^{\prime}:=\left(\begin{array}{ccc}
\left(1+h^{2} O(h)\right) & h^{2}(1+O(h)) & h^{2}(1+O(h)) \\
h O(h) & (1+h(1+O(h))) & h(1+O(h)) \\
O(h) & (1+O(h)) & (1+O(h))
\end{array}\right), \\
R_{n}^{\prime}:=\left(\begin{array}{c}
h^{2} \int_{0}^{1} \int_{0}^{s}\left(\sum_{j=1}^{m} L_{j}(\tau) R_{n, j}\right) d \tau d s \\
h \int_{0}^{1}\left(\sum_{j=1}^{m} L_{j}(\tau) R_{n, j}\right) d \tau \\
\sum_{j=1}^{m} L_{j}(1) R_{n, j}
\end{array}\right) .
\end{array}
$$

Equation (4.13) has the same form as (2.11). Since $h=0$ implies that the matrix $M^{\prime}$ has eigenvalues $\lambda_{1}^{\prime}=\lambda_{2}^{\prime}=\lambda_{3}^{\prime}=1$, we can prove the following theorem. 


\section{Stability of the collocation methods}

Theorem 4.1. For every choice of the collocation parameters $\left\{c_{j}\right\}_{j=1, \ldots, m}$, an $(m, 0)$-method is stable for all $m \geq 1$.

\section{Stability of $S_{m+1}^{(1)}\left(Z_{n}\right)$}

In this section, we will investigate the stability when $d=1$.

From (2.5), the restriction of $u \in S_{m+1}^{(1)}\left(Z_{n}\right)$ to the subinterval $\sigma_{n}$ is given by

$$
\begin{aligned}
u(t)=u_{n}(t+\tau h)= & u_{n-1}\left(t_{n}\right)+u_{n-1}^{\prime}\left(t_{n}\right) \tau+\sum_{r=1}^{m} \beta_{n, r} \tau^{r+1}, \\
& \text { for } \tau \in(0,1], n=0,1, \ldots, N-1 .
\end{aligned}
$$

In this case, the collocation equation becomes

$$
V \beta_{n}=h^{2} W^{\prime \prime}\left(u_{n-1}\left(t_{n}\right), u_{n-1}^{\prime}\left(t_{n}\right), u_{n-1}^{\prime \prime}\left(t_{n}\right)\right)^{T}+h^{2} R_{n},
$$

for $n=0,1 \ldots, N-1$, where $W^{\prime \prime}$ is the $m \times 3$ matrix whose elements are

$$
\left(W^{\prime \prime}\right)_{j, r}:= \begin{cases}\nu h c_{j} & \text { if } r=1, \\ c_{j} h\left(\alpha_{0}+\frac{\nu h c_{j}}{2}\right) & \text { if } r=2, \\ 1 & \text { if } r=3\end{cases}
$$

and the matrix $V$ and the vector $R_{n}$ are defined in (2.6) when $d=1$.

Using the same procedure as in Section 4, we can derive the system

$$
\left(\begin{array}{l}
u_{n}\left(t_{n+1}\right) \\
u_{n}^{\prime}\left(t_{n+1}\right) \\
u_{n}^{\prime \prime}\left(t_{n+1}\right)
\end{array}\right)=M^{\prime \prime}\left(\begin{array}{l}
u_{n-1}\left(t_{n}\right) \\
u_{n-1}^{\prime}\left(t_{n}\right) \\
u_{n-1}^{\prime \prime}\left(t_{n}\right)
\end{array}\right)+(1+O(h)) R_{n}^{\prime \prime}, \quad \text { for } n=0,1, \ldots, N-1,
$$

where

$$
\begin{gathered}
M^{\prime \prime}:=\left(\begin{array}{ccc}
1+h^{2} O(h) & h^{2} O(h) & h^{2}(1+O(h)) \\
h O(h) & 1+h O(h) & h(1+O(h)) \\
O(h) & O(h) & 1+O(h)
\end{array}\right), \\
R_{n}^{\prime \prime}:=\left(\begin{array}{c}
h^{2} \int_{0}^{1} \int_{0}^{s}\left(\sum_{j=1}^{m} L_{j}(\tau) R_{n, j}\right) d \tau d s \\
h \int_{0}^{1}\left(\sum_{j=1}^{m} L_{j}(\tau) R_{n, j}\right) d \tau \\
\sum_{j=1}^{m} L_{j}(1) R_{n, j}
\end{array}\right) .
\end{gathered}
$$

Equation (5.4) has the same form as (2.11). Since $h=0$ implies that the matrix $M^{\prime \prime}$ has eigenvalues $\lambda_{1}^{\prime}=\lambda_{2}^{\prime}=\lambda_{3}^{\prime}=1$, we can prove the following theorem. 
Edris Rawashdeh et al. 1063

Theorem 5.1. For every choice of the collocation parameters $\left\{c_{j}\right\}_{j=1, \ldots, m}$, an $(m, 1)$-method is stable for all $m \geq 1$.

\section{Numerical examples}

The $(3, d)$-method is tested using the following three examples in the interval $[0,1]$ with step size $h=0.05$. The following notations will be used in the presentation.

$$
e_{1}:=\left|y\left(t_{1}\right)-u\left(t_{1}\right)\right|, \quad e_{N / 2}:=|y(0.5)-u(0.5)|, \quad e_{N}:=|y(1)-u(1)|,
$$

where $u \in S_{3+d}^{d}\left(Z_{n}\right)$ is the approximate solution.

Example 6.1. Consider the following integrodifferential equation of second order:

$$
y^{\prime \prime}(t)=1+\frac{1}{2} y(t)+\frac{1}{2} \int_{0}^{t} y(s) d s, \quad y(0)=2, \quad y^{\prime}(0)=2,
$$

where $y(t)=2 e^{t}$ is the exact solution.

Example 6.2. Consider the following integrodifferential equation of second order:

$$
y^{\prime \prime}(t)=q(t)-\frac{t^{2}}{16} y^{\prime}(t)+\int_{0}^{t} t^{2} s y^{\prime}(s) d s, \quad y(0)=1, \quad y^{\prime}(0)=4,
$$

where $q(t)$ is chosen so that $y(t)=\sin 4 t$ is the exact solution.

Example 6.3. Consider the following integrodifferential equation of second order:

$$
\begin{aligned}
y^{\prime \prime}(t)= & q(t)+p_{1}(t) y(t)+p_{2}(t) y^{\prime}(t)+\int_{0}^{t} y(s) d s \\
& +\int_{0}^{t} t s^{2} y^{\prime}(s) d s, \quad y(0)=2, \quad y^{\prime}(0)=0,
\end{aligned}
$$

with

$$
p_{1}(t)=-t^{3}+2 t-1, \quad p_{2}(t)=1-2 t^{2},
$$

where $q(t)$ is chosen so that $y(t)=1+\cos t$ is the exact solution.

(a) If the collocation parameters are uniformly distributed, that is, $c_{1}=1 / 3, c_{2}=2 / 3$, and $c_{3}=1$, then we have Tables 6.1, 6.2, and 6.3 corresponding to Examples 6.1, 6.2 , and 6.3 , respectively.

(b) If the collocation parameters are the Radau II points, that is, $c_{1}=(4-\sqrt{6}) / 10$, $c_{2}=(4+\sqrt{6}) / 10$, and $c_{3}=1$, then we have Tables $6.4,6.5$, and 6.6 corresponding to Examples 6.1, 6.2, and 6.3, respectively.

(c) If the collocation parameters are the Gauss points, that is, $c_{1}=(5-\sqrt{15}) / 10, c_{2}=$ $1 / 2$, and $c_{3}=(5+\sqrt{15}) / 10$, then we have Tables $6.7,6.8$, and 6.9 corresponding to Examples 6.1, 6.2, and 6.3, respectively.

(d) If the first two collocation parameters are the Gauss points, that is, $c_{1}=(3-$ $\sqrt{3}) / 6, c_{2}=(3+\sqrt{3}) / 6$, and $c_{3}=1$, then we have Tables $6.10,6.11$, and 6.12 corresponding to Examples 6.1, 6.2, and 6.3, respectively. 
1064 Stability of the collocation methods

Table 6.1. Approximate error for Example 6.1.

\begin{tabular}{cccc}
\hline$d$ & $e_{1}$ & $e_{N / 2}$ & $e_{N}$ \\
\hline 2 & 0 & 0 & $1.00 \times 10^{-7}$ \\
3 & 0 & $1.00 \times 10^{-9}$ & $3.00 \times 10^{-9}$ \\
4 & 0 & $2.40 \times 10^{10}$ & $1.46 \times 10^{38}$ \\
\hline
\end{tabular}

Table 6.2. Approximate error for Example 6.2.

\begin{tabular}{cccc}
\hline$d$ & $e_{1}$ & $e_{N / 2}$ & $e_{N}$ \\
\hline 2 & $1.3 \times 10^{-9}$ & $3.32 \times 10^{-4}$ & $2.10 \times 10^{-3}$ \\
3 & $3.00 \times 10^{-10}$ & $3.32 \times 10^{-4}$ & $2.11 \times 10^{-3}$ \\
4 & $3.00 \times 10^{-10}$ & $4.20 \times 10^{13}$ & $2.56 \times 10^{41}$ \\
\hline
\end{tabular}

Table 6.3. Approximate error for Example 6.3.

\begin{tabular}{cccc}
\hline$d$ & $e_{1}$ & $e_{N / 2}$ & $e_{N}$ \\
\hline 2 & 0 & $8.36 \times 10^{-6}$ & $1.04 \times 10^{-3}$ \\
3 & $1.00 \times 10^{-10}$ & $8.35 \times 10^{-6}$ & $1.04 \times 10^{-3}$ \\
4 & 0 & $2.02 \times 10^{12}$ & $1.24 \times 10^{40}$ \\
\hline
\end{tabular}

Table 6.4. Approximate error for Example 6.1.

\begin{tabular}{cccc}
\hline$d$ & $e_{1}$ & $e_{N / 2}$ & $e_{N}$ \\
\hline 2 & 0 & 0 & $7.00 \times 10^{-9}$ \\
3 & 0 & $7.35 \times 10^{-4}$ & $6.23 \times 10^{8}$ \\
4 & 0 & $8.02 \times 10^{23}$ & $7.81 \times 10^{65}$ \\
\hline
\end{tabular}

Table 6.5. Approximate error for Example 6.2.

\begin{tabular}{cccc}
\hline$d$ & $e_{1}$ & $e_{N / 2}$ & $e_{N}$ \\
\hline 2 & $1.40 \times 10^{-9}$ & $3.32 \times 10^{-4}$ & $2.1 \times 10^{-3}$ \\
3 & $2.00 \times 10^{-10}$ & $4.52 \times 10^{-2}$ & $3.87 \times 10^{10}$ \\
4 & $8.00 \times 10^{-10}$ & $5.61 \times 10^{27}$ & $5.48 \times 10^{69}$ \\
\hline
\end{tabular}

Table 6.6. Approximate error for Example 6.3.

\begin{tabular}{cccc}
\hline$d$ & $e_{1}$ & $e_{N / 2}$ & $e_{N}$ \\
\hline 2 & 0 & $8.36 \times 10^{-6}$ & $1.04 \times 10^{-3}$ \\
3 & $1.00 \times 10^{-11}$ & $8.55 \times 10^{-3}$ & $7.34 \times 10^{9}$ \\
4 & $3.00 \times 10^{-10}$ & $2.34 \times 10^{27}$ & $2.28 \times 10^{69}$ \\
\hline
\end{tabular}

From these numerical examples, we observe that a $(3, d)$-method is stable for $d=2$ and it is unstable for $d=4$. In the case $d=3$, this method is stable if the collocation parameters are uniformly distributed (i.e., $c_{j}:=j / 3$, for $\left.j=1,2,3\right)$ as in case a, or $c_{1}=(3-\sqrt{3}) / 6$, 
Edris Rawashdeh et al. 1065

Table 6.7. Approximate error for Example 6.1.

\begin{tabular}{cccc}
\hline$d$ & $e_{1}$ & $e_{N / 2}$ & $e_{N}$ \\
\hline 2 & $1.55 \times 10^{-6}$ & $2.55 \times 10^{-3}$ & $2.46 \times 10^{-2}$ \\
3 & $9.30 \times 10^{-6}$ & $7.84 \times 10^{20}$ & $1.80 \times 10^{56}$ \\
4 & $1.01 \times 10^{-4}$ & $5.42 \times 10^{52}$ & $5.03 \times 10^{116}$ \\
\hline
\end{tabular}

Table 6.8. Approximate error for Example 6.2.

\begin{tabular}{cccc}
\hline$d$ & $e_{1}$ & $e_{N / 2}$ & $e_{N}$ \\
\hline 2 & $1.6 \times 10^{-9}$ & $3.59 \times 10^{-4}$ & $1.00 \times 10^{-4}$ \\
3 & $1.10 \times 10^{-9}$ & $4.19 \times 10^{17}$ & $3.78 \times 10^{53}$ \\
4 & $9.40 \times 10^{-9}$ & $4.80 \times 10^{48}$ & $4.47 \times 10^{112}$ \\
\hline
\end{tabular}

Table 6.9. Approximate error for Example 6.3.

\begin{tabular}{cccc}
\hline$d$ & $e_{1}$ & $e_{N / 2}$ & $e_{N}$ \\
\hline 2 & 0 & $4.83 \times 10^{-3}$ & $3.65 \times 10^{-2}$ \\
3 & 0 & $3.40 \times 10^{23}$ & $1.44 \times 10^{59}$ \\
4 & $2.00 \times 10^{-4}$ & $9.96 \times 10^{52}$ & $9.40 \times 10^{116}$ \\
\hline
\end{tabular}

Table 6.10. Approximate error for Example 6.1.

\begin{tabular}{cccc}
\hline$d$ & $e_{1}$ & $e_{N / 2}$ & $e_{N}$ \\
\hline 2 & $1.00 \times 10^{-9}$ & $4.00 \times 10^{-9}$ & $1.20 \times 10^{-8}$ \\
3 & 0 & 0 & $7.00 \times 10^{-9}$ \\
4 & 0 & $4.45 \times 10^{13}$ & $5.12 \times 10^{43}$ \\
\hline
\end{tabular}

Table 6.11. Approximate error for Example 6.2.

\begin{tabular}{cccc}
\hline$d$ & $e_{1}$ & $e_{N / 2}$ & $e_{N}$ \\
\hline 2 & $1.10 \times 10^{-9}$ & $3.32 \times 10^{-4}$ & $2.11 \times 10^{-3}$ \\
3 & $6.00 \times 10^{-10}$ & $3.32 \times 10^{-4}$ & $2.11 \times 10^{-3}$ \\
4 & $3.00 \times 10^{-10}$ & $5.43 \times 10^{16}$ & $6.26 \times 10^{46}$ \\
\hline
\end{tabular}

Table 6.12. Approximate error for Example 6.3.

\begin{tabular}{cccc}
\hline$d$ & $e_{1}$ & $e_{N / 2}$ & $e_{N}$ \\
\hline 2 & 0 & $8.36 \times 10^{-6}$ & $1.04 \times 10^{-3}$ \\
3 & $1.00 \times 10^{-10}$ & $8.36 \times 10^{-6}$ & $1.04 \times 10^{-3}$ \\
4 & $1.00 \times 10^{-10}$ & $3.06 \times 10^{15}$ & $3.54 \times 10^{45}$ \\
\hline
\end{tabular}

$c_{2}=(3+\sqrt{3}) / 6$, and $c_{3}=1$ as in case $(\mathrm{d})$. These examples illustrate the conclusions of Theorem 5.1. 


\section{References}

[1] H. Brunner, A survey of recent advances in the numerical treatment of Volterra integral and integro-differential equations, J. Comput. Appl. Math. 8 (1982), no. 3, 213-229.

[2] _ Polynomial spline collocation methods for Volterra integrodifferential equations with weakly singular kernels, IMA J. Numer. Anal. 6 (1986), no. 2, 221-239.

[3] W The approximate solution of initial-value problems for general Volterra integrodifferential equations, Computing 40 (1988), no. 2, 125-137.

[4] H. Brunner and J. D. Lambert, Stability of numerical methods for Volterra integro-differential equations, Computing 12 (1974), no. 1, 75-89.

[5] H. Brunner, A. Pedas, and G. Vainikko, Piecewise polynomial collocation methods for linear Volterra integro-differential equations with weakly singular kernels, SIAM J. Numer. Anal. 39 (2001), no. 3, 957-982.

[6] H. Brunner and P. J. van der Houwen, The Numerical Solution of Volterra Equations, CWI Monographs, vol. 3, North-Holland Publishing, Amsterdam, 1986.

[7] I. Danciu, Numerical stability of collocation methods for Volterra integro-differential equations, Rev. Anal. Numér. Théor. Approx. 26 (1997), no. 1-2, 59-74.

[8] M. E. A. El-Tom, On the numerical stability of spline function approximations to solutions of Volterra integral equations of the second kind, Nordisk Tidskr. Informationsbehandling (BIT) 14 (1974), 136-143.

[9] A. Goldfine, Taylor series methods for the solution of Volterra integral and integro-differential equations, Math. Comp. 31 (1977), no. 139, 691-707.

[10] D. Harvey, Global approximations of initial value problems for speical second order ordinary differential equation, M. S. thesis, Dalhousie University, Halifax, Nova Scotia, 1971.

[11] M. Miculá and G. Micula, Sur la résolution numérique des équations intégrales du type de Volterra de seconde espèce à l'aide des fonctions splines, Studia Univ. Babeș-Bolyai Ser. Math.Mech. 18 (1973), no. 2, 65-68 (French).

[12] T. Lin, Y. Lin, M. Rao, and S. Zhang, Petrov-Galerkin methods for linear Volterra integrodifferential equations, SIAM J. Numer. Anal. 38 (2000), no. 3, 937-963.

[13] V. Volterra, Theory of Functionals and of Integral and Integro-Differential Equations, Dover Publications, New York, 1959.

Edris Rawashdeh: Department of Mathematics, Center for Applied Mathematics and Polymer Fluid Dynamics, Central Michigan University, Mount Pleasant, MI 48859, USA

E-mail address: edirash@yahoo.com

Dave McDowell: Department of Mathematics, Center for Applied Mathematics and Polymer Fluid Dynamics, Central Michigan University, Mount Pleasant, MI 48859, USA

E-mail address: mcdow1dg@cmich.edu

Leela Rakesh: Department of Mathematics, Center for Applied Mathematics and Polymer Fluid Dynamics, Central Michigan University, Mount Pleasant, MI 48859, USA

E-mail address: leela.rakesh@cmich.edu 


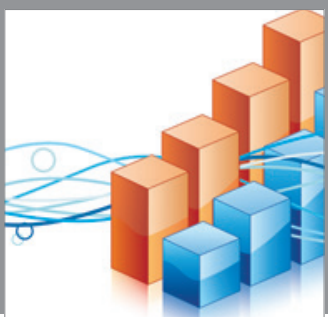

Advances in

Operations Research

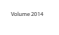

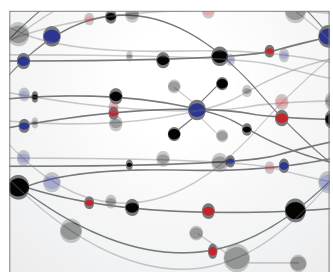

\section{The Scientific} World Journal
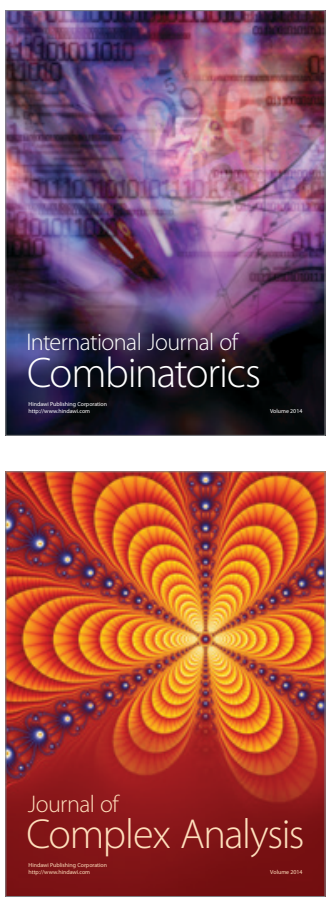

International Journal of

Mathematics and

Mathematical

Sciences
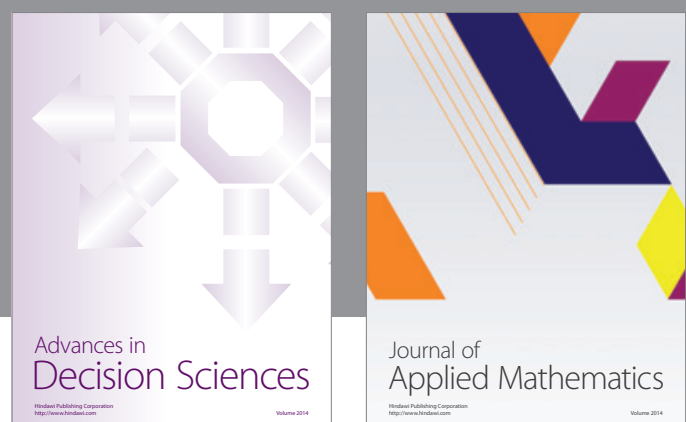

Journal of

Applied Mathematics
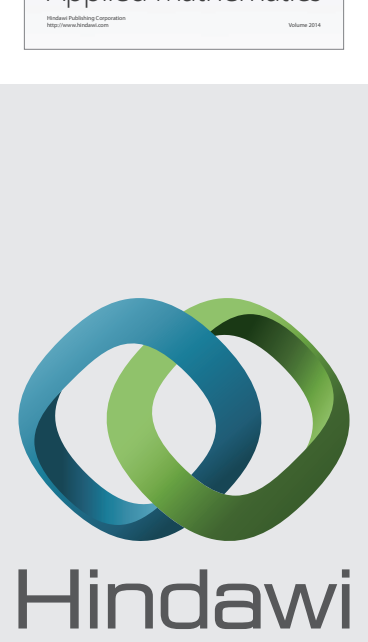

Submit your manuscripts at http://www.hindawi.com
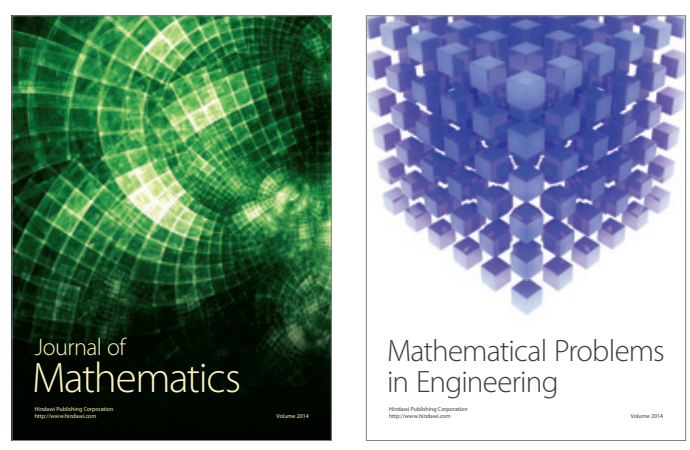

Mathematical Problems in Engineering
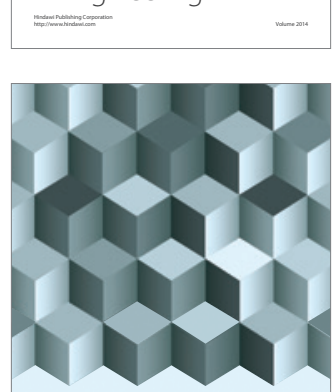

Journal of

Function Spaces
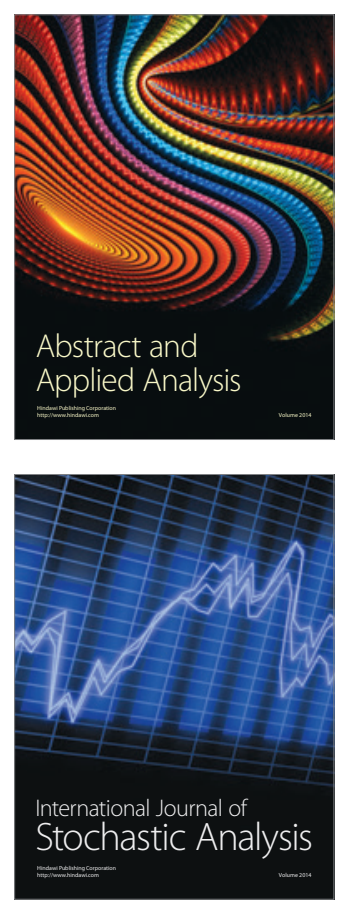

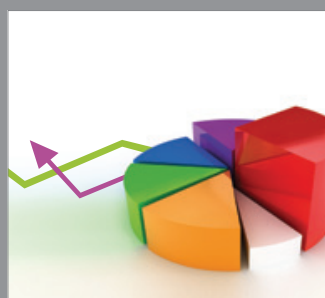

ournal of

Probability and Statistics

Promensencen
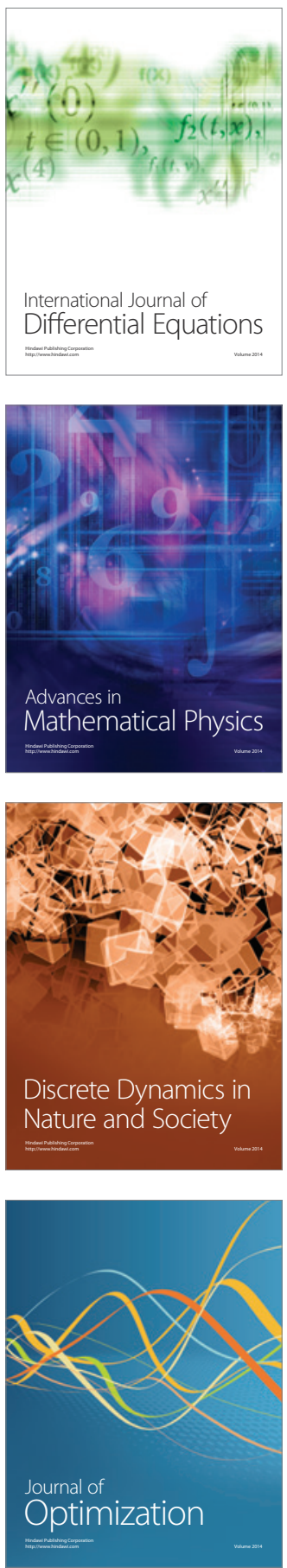